\title{
ELEMENTS OF NON-RELATIVISTIC QUANTUM MECHANICS
}


This page is intentionally left blank 


\section{ELEMENTS OF \\ NON-RELATIVISTIC \\ QUANTUM MECHANICS}

\section{Luis Sobrino \\ University of British Columbia, Canada}




\section{Published by}

World Scientific Publishing Co Pte Ltd

P O Box 128, Farrer Road, Singapore 912805

USA office: Suite 1B, 1060 Main Street, River Edge, NJ 07661

UK office: 57 Shelton Street, Covent Garden, London WC2H 9HE

\section{Library of Congress Cataloging-in-Publication Data}

Sobrino, Luis.

Elements of non-relativistic quantum mechanics / Luis Sobrino.

p. $\mathrm{cm}$.

Includes bibliographical references and index.

ISBN 9810223862

1. Nonrelativistic quantum mechanics. I. Title.

QC174.24.N64S57 1996

$530.12-\mathrm{dc} 20$

96-3188

CIP

\section{British Library Cataloguing-in-Publication Data}

A catalogue record for this book is available from the British Library.

Copyright 1996 by World Scientific Publishing Co. Pte. Ltd.

All rights reserved. This book, or parts thereof, may not be reproduced in any form or by any means, electronic or mechanical, including photocopying, recording or any information storage and retrieval system now known or to be invented, without written permission from the Publisher.

For photocopying of material in this volume, please pay a copying fee through the Copyright Clearance Center, Inc., 222 Rosewood Drive, Danvers, MA 01923, USA.

Printed in Singapore. 


\section{PREFACE}

It is almost a trivial observation that many collective endeavours require the accomplishment of numerous complicated tasks, and that the different individuals that perform them may lose sight of the overall design. Like other creative endeavours, the scientific quest is particularly prone to such a state of affairs, for not only does the difficulty of the tasks absorb the mind but the beauty of what is learnt in performing them engages the soul. Like a diver, who may be so charmed by the nearby coral so as to forget that its beauty is but a minute reflection of the majestic beauty of the ocean, a spectroscopist may be so taken by the beautiful symmetries that the molecules exhibit so as to loose sight of the wonderful structure of quantum mechanics that informs them.

For many years, I have taught a graduate course on non-relativistic quantum mechanics at the University of British Columbia. As a first graduate course in the subject, it was to provide a good understanding of the principles as well as the necessary basic skills for the application of quantum mechanics to a diversity of fields. The students, coming, as the did, from universities all over the world, had very diverse backgrounds and I could not assume that every one of them had a good grasp of the basic principles of quantum mechanics, all I could assume was that they all had studied the standard elementary applications. Because the course was required of all graduate students in Physics, the prospective fields of its audience ranged widely - from quantum field theory to physical oceanography -, which meant that I could not maintain their interest by centring the lectures on any particular field of application. Faced with these constraints, I felt that the best way of achieving the aims of the course was to present the theory of quantum mechanics taking care to exhibit its coherence and beauty, hoping to maintain the students' interest by appealing to their aesthetic sense. Now that I am nearing the end of 
my teaching career, I feel that the approach taken was successful, and I have the satisfaction of knowing that this feeling is shared by many of my former students.

The present book was born of the experience gained in teaching the course just described. It is not meant to be a textbook for such a course. It covers less material than the course did, but treated in more depth. As a first approximation, the book might be described as a coherent presentation of the core of the course augmented by the answers to questions that many of my students and some of my colleagues have put to me in the course of the years. Many of these questions I could not answer by reference to the literature, for the available answers were presented at a level not accessible to those that formulated them.

In spite of the suggestions that I received from students and colleagues I would not have undertaken the task of writing this book had it not been for my own desire to have a comprehensive overview of quantum mechanical theory and gain a better appreciation of its beauty. I feel now that the exercise was worth the effort and I hope that the reader would gain as much aesthetic satisfaction from reading this book as the author gained from writing it.

To limit the size of the book, I had to decide what not to include. It was clear to me that those problems which are solved in detail in every textbook (e.g., the hydrogen atom) could be left out, unless they were necessary for the development of the presentation (such as the harmonic oscillator) or particularly useful as illustrations of the theory. More difficult was the decision not to include approximate methods and scattering theory because, although these two subjects have in some sense an ancillary status within the general theory, not only are they of essential importance in the applications but they also have had an enormous influence in the way we think about the physical world. Even more difficult was the decision not to treat the quantization of fields more extensively and just to touch it very briefly in connection with the study of an assembly of particles.

I had no difficulty in deciding not to include a discussion of the interpretations of quantum mechanics nor of the closely related problem of measurement. These extremely interesting questions lie in a "metalevel" above quantum theory and, in my opinion, would require a full volume to be properly discussed.

What is included constitutes the core of non-relativistic quantum-mechanical theory, the elements needed to properly understand the developments of the subject and its applications.

As can be gathered from the introductory paragraphs of this preface, these elements are presented with a different emphasis than is common in the standard textbooks, and at a higher level, but without requiring from the reader more than a working knowledge of quantum mechanics on the physical side, and the mathemat- 
ical background in algebra and analysis normally acquired at the undergraduate level. This book occupies a largely empty niche between the usual textbooks and the more mathematically advanced writings on the subject. I expect it would be of interest both to graduate students and to many practising scientists who would like to delve a little into the structure of quantum mechanics and into the mathematical notions that underlie it.

Quantum systems cannot be described in terms of our sensory experience, to understand them it is necessary to use the abstract language provided by the algebra of linear operators on a Hilbert space. For this reason, three mathematical appendices have been included. For ease of reference, their paragraphs are numbered and are often referred to in the main text. Appendix A reviews finitedimensional vector spaces; the necessary results in the theory of Hilbert spaces are summarized in Appendix B, while Appendix C presents some elements of the theory of distributions leading to an explanation of the mathematical basis of Dirac's formalism. The first four chapters of the book make use of the material in sections 1 to 3 of Appendix A which are mostly intended to serve as a reminder. Before embarking in a thorough reading of the rest of the book, the reader is invited to peruse the final sections of Appendix $A$ as well as appendices $B$ and $C$. A fourth appendix, D, includes some pertinent results in classical mechanics.

Group theoretical considerations pertaining mostly to the Galilei and to the symmetric groups play an important part in the exposition. It has been possible to develop them without assuming on the part of the reader much more than a knowledge of the definition of group. They are not included in the appendices but in appropriate places in the main body of the text.

A remark on the mathematical aspects of the presentation is in order here. Our experimental instruments are finite, in size, in energy, and in the number of configurations that they can exhibit. This implies that, in principle, only the language of finite-dimensional vector spaces is needed to explain experimental results and to understand the structure of quantum mechanics. However, it is extremely fruitful - and necessary if we want to embed the theory in a space and time continuum - to consider idealized instruments capable of an infinite number of configurations, which require a description cast in the language of infinite-dimensional spaces. Because these infinite instruments are approximations of the actual finite ones, physicists have all reason to ignore those properties of the infinite-dimensional Hilbert space that cannot be obtained from the properties of finite-dimensional ones by some, not necessarily unique, physically based limiting procedure. A working knowledge of the mathematical description that results from the adopted limiting procedure is necessary to understand many of the developments of quantum mechanics. The mathematical presentation reflects these considerations: the results 
pertaining to finite-dimensional spaces, necessary for the understanding of the structure of quantum mechanics, are presented with thoroughness; their generalizations to infinite-dimensional spaces are discussed in less detail, although references to the mathematical literature are given for the benefit of the interested reader.

After an introductory chapter that sets the point of view adopted in the exposition, the main body of the book can be thought of as being divided into two parts. In the first one, Chapters II to IV, are presented and elaborated the basic rules (I prefer this word to "postulates" or "axioms" because it does not imply a mathematical rigour that the exposition does not have) as they apply to model systems describable in the language of finite-dimensional spaces. In this way one can exhibit the basic structure of the theory unencumbered by the mathematical complications that arise in infinite-dimensional spaces.

The second part deals with the generalization of the theory and its applications to different types of systems. The general theory is presented in Chapter $\mathrm{V}$, in which the concept of kinematical symmetries is also introduced and applied to those symmetries that arise from galilean invariance. Chapters VI and VII which discuss the particle without internal degrees of freedom are followed by the study in Chapter VIII of the dynamical symmetries of quantum mechanics; at this point the reader may find it helpful to review the classical results of Appendix D. The particle with spin is discussed in Chapter IX, and composite systems in the remaining two chapters. The connection between galilean invariance and the fundamental observables of a system constitutes the unifying thread that runs through this part of the book.

Notes, mainly but not exclusively bibliographical, to most sections can be found after the appendices, followed by a selected bibliography.

A standard nomenclature has been adopted; minor exceptions are the use of "density operator" in preference to the more generally used "density matrix", of "commensurable observables" that seems more appropriate than "compatible" or "simultaneously measurable observables" and of "maximal observable" instead of the lengthier "complete set of commuting observables".

Dirac's formalism and notation is used throughout the main body of the book. From the mathematical point of view, however, this notation has some disadvantages; for this reason and because the use of Dirac's notation is not universal, the main results of the mathematical appendices are given in ordinary notation. Each section of these appendices ends with an explanation of the connection between the two notations.

For reference, mathematical expressions within a chapter are numbered in the common decimal system. Within each chapter, they are referred to by their number 
[e.g., (3.5)]. In the references across chapters the expression number is preceded by the chapter number [e.g, (II.3.5)]. Paragraphs in the appendices are also numbered in the decimal system (but not enclosed in parentheses) and referred to in a similar manner (e.g., paragraph 3.2.2 of Appendix B will be referred to as 3.2 .2 from within the appendix and as B.3.2.2 from without). Mathematical expressions in the appendices are not numbered.

I owe a debt of gratitude to all those that made possible the writing of this book. I am particularly indebted to the many former students whose comments and questions contributed so much to my understanding of quantum mechanics. Among them, I would dare to name Ulrike Närger, Steve Patitsas, Mark Shegelski and Mike Sofer, who collaborated with me in the teaching of the subject. My thanks are also due to all those colleagues with whom I have discussed many of the topics included in this book and, especially, to F.W. Dalby whose provocative questions always demanded an answer. My readings, of course, influenced the shaping of my course and are reflected in my writing. Two books I should mention in this connection: Messiah's Quantum Mechanics and Jauch's Foundations of Quantum Mechanics. To their authors I express my gratitude.

In the latter stages of the preparation of this book, I received valuable comments from Mark MacLean, who kindly agreed to read the mathematical appendices, and essential technical help from Janet Clark, who typeset the manuscript in $T_{E} X$. To both of them, my sincere thanks.

In concluding this preface, I must ask the reader to forgive the dryness of my style, for the language in which I am writing is not the language in which I learned to laugh and to cry.

Vancouver, April 1995. 
This page is intentionally left blank 


\section{CONTENTS}

PREFACE v v

I. INTRODUCTION

1. Description of experiments 1

2. The concept of physical system 3

3. Preparation and measurement 6

II. STATES AND OBSERVABLES 10

1. States and amplitudes 10

2. An example: spin $1 / 2 \quad 14$

3. Observables 20

4. The density operator 24

III. COMMENSURABILITY 30

1. The spin example continued 30

2. Different kinds of measurement 32

3. Commensurable observables 34

4. The uncertainty relation 38

$\begin{array}{ll}\text { IV. DYNAMICS } & \mathbf{4 0}\end{array}$

1. The dynamical postulate 40

2. Schrödinger, Heisenberg and mixed pictures 43

3. An aside on functions of several observables 47

4. The hamiltonian 50

5. Equations of motion in the different pictures 53

6. An example 58 
V. GENERAL FORMULATION 62

1. Generalization of the basic postulates 62

2. Dirac's formulation $\quad 65$

3. Kinematical symmetries 68

4. The dynamics under a kinematical symmetry 69

5. The galilean transformations as kinematical symmetries 72

6. The generators of kinematical galilean transformations 78

7. Superselection rules 86

VI. A PARTICLE IN ONE DIMENSION 89

1. A particle without spin 89

2. The momentum operator $\quad 92$

3. The velocity, the mass and the hamiltonian $\quad 94$

4. An example: the harmonic oscillator 98

5. A particle in a constant force field 102

VII. A SPINLESS PARTICLE IN THREE DIMENSIONS 106

1. Position, momentum and the hamiltonian 106

2. Rotations, the orbital angular momentum 110

3. The angular momentum commutation relations 112

4. Spectral resolution of the orbital angular momentum 116

5. The free particle 119

6. The Wigner distribution function 121

7. The harmonic oscillator 123

8. The spinless particle in a general coordinate system 125

9. The particle in a central field 130

VIII. DYNAMICAL SYMMETRIES AND CONSERVATION LAWS 133

1. The dynamical symmetry transformations of quantum mechanics 133

2. Unitary transformations and constants of the motion 135

3. Space inversion, the parity observable 139

4. Time reversal 142

5. The galilean transformations as dynamical symmetries 145

IX. A PARTICLE WITH SPIN 152

1. Internal degrees of freedom and galilean invariance 152

2. Spin and rotations 156 
3. The particle with spin $\quad 160$

4. The hamiltonian of a particle with spin 165

5. Example: a neutral particle in a magnetic field 171

X. SYSTEMS COMPOSED OF DIFFERENT SUBSYSTEMS 175

1. The description of systems formed by different subsystems $\quad 176$

$\begin{array}{ll}\text { 2. A system of two different particles } & 177\end{array}$

3. Some useful representations 186

4. The state of a subsystem 190

5. Example: states of a particle in a magnetic field 195

XI. SYSTEMS OF IDENTICAL PARTICLES 202

1. The description of systems formed by two identical subsystems 202

2. Example: a system of two identical particles 209

3. The description of systems of many identical particles 213

4. Number representation of systems of identical particles 222

5. An assembly of bosons 223

6. An assembly of fermions 230

APPENDIX A. FINITE-DIMENSIONAL VECTOR SPACES

1. Basic definitions 234

2. Operators in unitary spaces $\quad 237$

3. The spectral theorem 244

4. The tensor product 252

5. Antilinear operators 259

6. Wigner's theorem 260

APPENDIX B. THE HILBERT SPACE $\quad 264$

1. Basic definitions, unbounded operators 264

2. The spectral theorem $\quad 267$

3. Function space representation 278

APPENDIX C. DIRAC'S FORMULATION IN THE HILBERT SPACE 281

1. Tempered distributions 281

2. Generalization to an arbitrary Hilbert space 301

3. Dirac's formulation 303 
APPENDIX D. A REMINDER FROM CLASSICAL MECHANICS 312

1. Canonical transformations 312

2. A reminder from classical mechanics 316

$\begin{array}{ll}\text { NOTES } & 320\end{array}$

$\begin{array}{ll}\text { BIBLIOGRAPHY } & 325\end{array}$

$\begin{array}{ll}\text { INDEX } & 328\end{array}$ 